\title{
HILLE-KNESER-TYPE CRITERIA FOR SECOND-ORDER DYNAMIC EQUATIONS ON TIME SCALES
}

\author{
L. ERBE, A. PETERSON, AND S. H. SAKER \\ Received 31 January 2006; Revised 16 May 2006; Accepted 16 May 2006
}

We consider the pair of second-order dynamic equations, $\left(r(t)\left(x^{\Delta}\right)^{\gamma}\right)^{\Delta}+p(t) x^{\gamma}(t)=0$ and $\left(r(t)\left(x^{\Delta}\right)^{\gamma}\right)^{\Delta}+p(t) x^{\gamma \sigma}(t)=0$, on a time scale $\mathbb{T}$, where $\gamma>0$ is a quotient of odd positive integers. We establish some necessary and sufficient conditions for nonoscillation of Hille-Kneser type. Our results in the special case when $\mathbb{T}=\mathbb{R}$ involve the wellknown Hille-Kneser-type criteria of second-order linear differential equations established by Hille. For the case of the second-order half-linear differential equation, our results extend and improve some earlier results of Li and Yeh and are related to some work of Došlý and Řehák and some results of Řehák for half-linear equations on time scales. Several examples are considered to illustrate the main results.

Copyright $\odot 2006 \mathrm{~L}$. Erbe et al. This is an open access article distributed under the Creative Commons Attribution License, which permits unrestricted use, distribution, and reproduction in any medium, provided the original work is properly cited.

\section{Introduction}

The theory of time scales, which has recently received a lot of attention, was introduced by Stefan Hilger in his Ph.D. thesis in 1988 in order to unify continuous and discrete analysis, see [19]. This theory of "dynamic equations" unifies the theories of differential equations and difference equations, and also extends these classical cases to situations "in between," for example, to the so-called $q$-difference equations, and can be applied on different types of time scales. Many authors have expounded on various aspects of the new theory. A book on the subject of time scales, that is, measure chains, by Bohner and Peterson [5] summarizes and organizes much of time scale calculus for dynamic equations. For advances on dynamic equations on time scales, we refer the reader to the book by Bohner and Peterson [6].

In recent years, there has been an increasing interest in studying the oscillation of solutions of dynamic equations on time scales, which simultaneously treats the oscillation of the continuous and the discrete equations. In this way, we do not require to write the oscillation criteria for differential equations and then write the discrete analogues 
for difference equations. For convenience, we refer the reader to the results given in [1$4,7,8,10-18,20-33]$.

In this paper, we present some oscillation criteria of Hille-Kneser type for the secondorder dynamic equations of the form

$$
\begin{aligned}
& L_{1} x=\left(r(t)\left(x^{\Delta}(t)\right)^{\gamma}\right)^{\Delta}+p(t) x^{\gamma}(t)=0, \\
& L_{2} x=\left(r(t)\left(x^{\Delta}(t)\right)^{\gamma}\right)^{\Delta}+p(t) x^{\gamma \sigma}(t)=0,
\end{aligned}
$$

on an arbitrary time scale $\mathbb{T}$, where we assume throughout this paper that $r$ and $p$ are real $r d$-continuous functions on $\mathbb{T}$ with $r(t)>0, p(t)>0$, and $\gamma>0$ is a quotient of odd positive integers. We denote $x^{\sigma}:=x \circ \sigma$, where the forward jump operator $\sigma$ and the backward jump operator $\rho$ are defined by

$$
\sigma(t):=\inf \{s \in \mathbb{T}: s>t\}, \quad \rho(t):=\sup \{s \in \mathbb{T}: s<t\},
$$

where $\inf \varnothing:=\sup \mathbb{T}$ and $\sup \varnothing:=\inf \mathbb{T}$. A point $t \in \mathbb{T}$ is right-dense provided $t<\sup \mathbb{T}$ and $\sigma(t)=t$ and left-dense if $t>\inf \mathbb{T}$ and $\rho(t)=t$. A point $t \in \mathbb{T}$ is right-scattered provided $\sigma(t)>t$ and left-scattered if $\rho(t)<t$. By $x: \mathbb{T} \rightarrow \mathbb{R}$ is $r d$-continuous, we mean $x$ is continuous at all right-dense points $t \in \mathbb{T}$ and at all left-dense points $t \in \mathbb{T}$, left-hand limits exist (finite). The graininess function $\mu: \mathbb{T} \rightarrow \mathbb{R}^{+}$is defined by $\mu(t):=\sigma(t)-t$. Also $\mathbb{T}^{\kappa}:=\mathbb{T}-\{m\}$ if $\mathbb{T}$ has a left-scattered maximum $m$, otherwise, $\mathbb{T}^{\kappa}:=\mathbb{T}$.

Here the domain of $L_{1}$ and $L_{2}$ is defined by

$$
D=\left\{x: \mathbb{T} \longrightarrow \mathbb{R}:\left(r(t)\left(x^{\Delta}(t)\right)^{\gamma}\right)^{\Delta} \text { is } r d \text {-continous }\right\}
$$

When $\mathbb{T}=\mathbb{R}$, equations $L_{1} x=0$ and $L_{2} x=0$ are the half-linear differential equation

$$
\left(r(t)\left(x^{\prime}(t)\right)^{\gamma}\right)^{\prime}+p(t) x^{\gamma}(t)=0
$$

See the book by Došlý and Řehák [11] and the references there for numerous results concerning (1.5). When $\mathbb{T}=\mathbb{Z}, L_{1} x=0$ is the half-linear difference equation

$$
\Delta\left(r(t) \Delta(x(t))^{\gamma}\right)+p(t) x^{\gamma}(t)=0
$$

(in [9], the author studies the forced version of (1.6)). Also, If $\mathbb{T}=h \mathbb{Z}, h>0$, then $\sigma(t)=$ $t+h, \mu(t)=h$,

$$
y^{\Delta}(t)=\Delta_{h} y(t)=\frac{y(t+h)-y(t)}{h},
$$

and $L_{1} x=0$ becomes the generalized second-order half-linear difference equation

$$
\Delta_{h}\left(r(t) \Delta_{h}(x(t))^{\gamma}\right)+p(t) x^{\gamma}(t)=0
$$


If $\mathbb{T}=q^{\mathbb{N}}=\left\{t: t=q^{k}, k \in \mathbb{N}, q>1\right\}$, then $\sigma(t)=q t, \mu(t)=(q-1) t$,

$$
x^{\Delta}(t)=\Delta_{q} x(t)=\frac{x(q t)-x(t)}{(q-1) t}
$$

and $L_{1} x=0$ becomes the second-order half-linear $q$-difference equation

$$
\Delta_{q}\left(r(t) \Delta_{q}(x(t))^{\gamma}\right)+p(t) x^{\gamma}(t)=0
$$

If $\mathbb{N}_{0}^{2}=\left\{t^{2}: t \in \mathbb{N}_{0}\right\}$, then $\sigma(t)=(\sqrt{t}+1)^{2}$ and $\mu(t)=1+2 \sqrt{t}$,

$$
\Delta_{N} y(t)=\frac{y\left((\sqrt{t}+1)^{2}\right)-y(t)}{1+2 \sqrt{t}} \quad \text { for } t \in\left[t_{0}^{2}, \infty\right)
$$

and $L_{1} x=0$ becomes the second-order half-linear difference equation

$$
\Delta_{N}\left(r(t) \Delta_{N}(x(t))^{\gamma}\right)+p(t) x^{\gamma}(t)=0
$$

One may also write down the corresponding equations for $L_{2} x=0$ for the various time scales mentioned above. The terminology half linear arises because of the fact that the space of all solutions of $L_{1} x=0$ or $L_{2} x=0$ is homogeneous, but not generally additive. Thus, it has just "half" of the properties of a linear space. It is easily seen that if $x(t)$ is a solution of $L_{1} x=0$ or $L_{2} x=0$, then so also is $c x(t)$. We note that in some sense, much of the Sturmian theorey is valid for (1.2) but that is not the case for (1.1). We refer to Rehák [23] and to his Habilitation thesis [24] in which some open problems are also mentioned for (1.2).

Since we are interested in the asymptotic behavior of solutions, we will suppose that the time scale $\mathbb{T}$ under consideration is not bounded above, that is, it is a time scale interval of the form $[a, \infty)_{\mathbb{T}}:=[a, \infty) \cap \mathbb{T}$. Solutions vanishing in some neighborhood of infinity will be excluded from our consideration. A solution $x$ of $L_{i} x=0, i=1,2$, is said to be oscillatory if it is neither eventually positive nor eventually negative, otherwise, it is nonoscillatory. The equation $L_{i} x=0, i=1,2$, is said to be oscillatory if all its solutions are oscillatory. It should be noted that the essentials of Sturmian theory have been extended to the half-linear equation $L_{2} x=0$ (cf. Řehák [23]).

One of the important techniques used in studying oscillations of dynamic equations on time scales is the averaging function method. By means of this technique, some oscillation criteria for $L_{2} x=0$ for the case $\gamma=1$ have been established in [12] which involve the behavior of the integral of the coefficients $r$ and $p$. On the other hand, the oscillatory properties can be described by the so-called Reid roundabout theorem (cf. $[5,11,23])$. This theorem shows the connection among the concepts of disconjugacy, positive definiteness of the quadratic functional, and the solvability of the corresponding Riccati equation (or inequality) which in turn implies the existence of nonoscillatory solutions. The Reid roundabout theorem provides two powerful tools for the investigation of oscillatory properties, namely the Riccati technique and the variational principle.

Sun and Li [32] considered the half-linear second-order dynamic equation $L_{1} x=0$, where $\gamma \geq 1$ is an odd positive integer, and $r$ and $p$ are positive real-valued $r d$-continuous 
functions such that

$$
\int_{t_{0}}^{\infty}\left(\frac{1}{r(t)}\right)^{1 / \gamma} \Delta t=\infty
$$

and used the Riccati technique and Lebesgue's dominated convergence theorem to establish some necessary and sufficient conditions for existence of positive solutions.

For the oscillation of the second-order differential equation

$$
x^{\prime \prime}(t)+p(t) x(t)=0, \quad t \geq t_{0},
$$

Hille [20] extended Kneser's theorem and proved the following theorem (see also [31, Theorem B] and the reference cited therein).

Theorem 1.1 (Hille-Kneser-type criteria). Let

$$
p^{*}=\lim _{t \rightarrow \infty} \sup t^{2} p(t), \quad p_{*}=\lim _{t \rightarrow \infty} \inf t^{2} p(t) .
$$

Then (1.14) is oscillatory if $p_{*}>1 / 4$, and nonoscillatory if $p^{*}<1 / 4$. The equation can be either oscillatory or nonoscillatory if either $p_{*}$ or $p^{*}=1 / 4$.

So the following question arises: can one extend the Hille-Kneser theorem to the halflinear dynamic equations $L_{1} x=0$ and $L_{2} x=0$ on time scales, and from these deduce the oscillation and nonoscillation results for half-linear differential and difference equations? The main aim of this paper is to give an affirmative answer to this question concerning the nonoscillation result.

Our results in the special case when $\mathbb{T}=\mathbb{R}$ involve the results established by Li and Yeh [22], Kusano and Yoshida [21], and Yang [33] for the second-order half-linear differential equations, and when $r(t) \equiv 1$ and $\gamma=1$, the results involve the criteria of Hille-Kneser type for second-order differential equations established by Hille [20], and are new for (1.6)-(1.10). Also, in the special case, $\gamma=1$, we derive Hille-Kneser-type nonoscillation criteria for the second-order linear dynamic equation

$$
\left(r(t)\left(x^{\Delta}(t)\right)\right)^{\Delta}+p(t) x(t)=0,
$$

on a time scale $\mathbb{T}$, which are essentially new. Several examples are considered to illustrate the main results.

\section{Main results}

Our interest in this section is to establish some necessary and sufficient conditions of Hille-Kneser type for nonoscillation of $L_{1} x=0$ and $L_{2} x=0$ by using the Riccati technique. We search for a solution of the corresponding Riccati equations corresponding to $L_{1} x=0$ and $L_{2} x=0$, respectively. Associated with $L_{1} x=0$ is the Riccati dynamic equation

$$
R_{1} w=w^{\Delta}+p(t)+w^{\sigma} F(w, t)=0,
$$


L. Erbe et al. 5

where for $u \in \mathbb{R}$ and $t \in \mathbb{T}$,

$$
F(u, t)= \begin{cases}\frac{\left(1+\mu(t)(u / r(t))^{1 / \gamma}\right)^{\gamma}-1}{\mu(t)} & \text { if } \mu(t)>0, \\ \gamma\left(\frac{u}{r(t)}\right)^{1 / \gamma} & \text { if } \mu(t)=0 .\end{cases}
$$

Here we take the domain of the operator $R_{1}$ to be

$$
\mathbb{D}:=\left\{w: \mathbb{T} \longrightarrow \mathbb{R}: w^{\Delta} \text { is } r d \text {-continuous on } \mathbb{T}^{\kappa} \text { and }\left(\frac{w}{r}\right)^{1 / \gamma} \in \mathscr{R}\right\} \text {, }
$$

where $\mathscr{R}$ is the class of regressive functions [5, page 58] defined by

$$
\mathscr{R}:=\{x: \mathbb{T} \longrightarrow \mathbb{R}: x \text { is } r d \text {-continuous on } \mathbb{T} \text { and } 1+\mu(t) x(t) \neq 0\} .
$$

Associated with equation $L_{2} x=0$ is the Riccati dynamic equation

$$
R_{2} w=w^{\Delta}+p(t)+S(w, t)=0
$$

where for $u \in \mathbb{R}$ and $t \in \mathbb{T}$,

$$
S(u, t):= \begin{cases}u \frac{\left(1+\mu(t)(u / r(t))^{1 / \gamma}\right)^{\gamma}-1}{\mu(t)\left(1+\mu(t)(u / r(t))^{1 / \gamma}\right)^{\gamma}} & \text { if } \mu(t)>0, \\ \gamma u\left(\frac{u}{r(t)}\right)^{1 / \gamma} & \text { if } \mu(t)=0 .\end{cases}
$$

Here we take the domain of the operator $R_{2}$ to be $\mathbb{D}$. The dynamic Riccati equation (2.1) is studied in [32] (they assume $\gamma$ is an odd positive integer) and the Riccati dynamic equation (2.5) is studied extensively in [23]. A number of oscillation criteria are also given based on the variational technique. It is easy to show that if $w \in \mathbb{D}$, then $F(w(t), t)$ and $S(w(t), t)$ are $r d$-continuous on $\mathbb{T}$.

We next state two theorems that relate our second-order half-linear equations to their respective Riccati equations.

Theorem 2.1 (factorization of $L_{1}$ ). If $x \in D$ with $x(t) \neq 0$ on $\mathbb{T}$ and $w(t):=r(t)\left(x^{\Delta}(t)\right)^{\gamma /}$ $x^{\gamma}(t), t \in \mathbb{T}^{\kappa}$, then $w \in \mathbb{D}$ and

$$
L_{1} x(t)=x^{\gamma}(t) R_{1} w(t), \quad t \in \mathbb{T}^{\kappa^{2}} .
$$

Conversely, if $w \in \mathbb{D}$ and

$$
x(t):=e_{(w / r)^{1 / \gamma}}\left(t, t_{0}\right),
$$

then $x \in D, x(t) \neq 0$, and (2.7) holds. Furthermore, $x(t) x^{\sigma}(t)>0$ if and only if $(w / r)^{1 / \gamma} \in$ $\mathscr{R}^{+}:=\{x \in \mathscr{R}: 1+\mu(t) x(t)>0, t \in \mathbb{T}\}$. 
Proof. First we prove the converse statement. Let $w \in \mathbb{D}$, then since $(w / r)^{1 / \gamma} \in \mathscr{R}$, we know that

$$
x(t)=e_{(w / r)^{1 / \gamma}}\left(t, t_{0}\right) \neq 0
$$

is well defined (see [5, page 59]). Let $x(t)=e_{(w / r)^{1 / \gamma}}\left(t, t_{0}\right)$, then $x^{\Delta}(t)=(w(t) / r(t))^{1 / \gamma} x(t)$ from which it follows that

$$
r(t)\left(x^{\Delta}(t)\right)^{\gamma}=x^{\gamma}(t) w(t) .
$$

From this last equation and the product rule, we get that

$$
\begin{aligned}
L_{1} x(t) & =\left(r(t)\left(\left(x^{\Delta}(t)\right)^{\gamma}\right)^{\Delta}+p(t) x^{\gamma}(t)=x^{\gamma}(t) w^{\Delta}(t)+w^{\sigma}(t)\left(x^{\gamma}\right)^{\Delta}(t)+p(t) x^{\gamma}(t)\right. \\
& =x^{\gamma}(t)\left[w^{\Delta}(t)+\frac{\left(x^{\gamma}\right)^{\Delta}(t)}{x^{\gamma}(t)} w^{\sigma}(t)+p(t)\right] .
\end{aligned}
$$

We now show that

$$
\frac{\left(x^{\gamma}\right)^{\Delta}(t)}{x^{\gamma}(t)}=F(w(t), t)
$$

First if $\mu(t)=0$, then

$$
\left(x^{\gamma}\right)^{\Delta}(t)=\gamma x^{\gamma-1} x^{\Delta}(t)
$$

from which it follows that

$$
\frac{\left(x^{\gamma}\right)^{\Delta}(t)}{x^{\gamma}(t)}=\gamma \frac{x^{\Delta}(t)}{x(t)}=\gamma\left(\frac{w(t)}{r(t)}\right)^{1 / \gamma}=F(w(t), t) .
$$

Next assume $\mu(t)>0$, then

$$
\begin{aligned}
\frac{\left(x^{\gamma}\right)^{\Delta}(t)}{x^{\gamma}(t)} & =\frac{x^{\gamma}(\sigma(t))-x^{\gamma}(t)}{\mu(t) x^{\gamma}(t)}=\frac{\left(x^{\sigma}(t) / x(t)\right)^{\gamma}-1}{\mu(t)}=\frac{\left(1+\mu(t)\left(x^{\Delta}(t) / x(t)\right)\right)^{\gamma}-1}{\mu(t)} \\
& =\frac{\left(1+\mu(t)(w(t) / r(t))^{1 / \gamma}\right)^{\gamma}-1}{\mu(t)}=F(w(t), t) .
\end{aligned}
$$

Hence in general we get that (2.12) holds. Using (2.11) and (2.12), we get the desired factorization $(2.7)$ in all cases.

Next assume $x \in D$ and $x(t) \neq 0$. Let $w(t)=r(t)\left(x^{\Delta}\right)^{\gamma}(t) / x^{\gamma}(t)$. Using the product rule

$$
w^{\Delta}(t)=\left(r(t)\left(x^{\Delta}(t)\right)^{\gamma}\right)^{\Delta} \frac{1}{x^{\gamma}(t)}+\left(r(t)\left(x^{\Delta}(t)\right)^{\gamma}\right)^{\sigma}\left(\frac{1}{x^{\gamma}(t)}\right)^{\Delta} .
$$


Hence

$$
x^{\gamma}(t) w^{\Delta}(t)=\left(r(t)\left(x^{\Delta}(t)\right)^{\gamma}\right)^{\Delta}+w^{\sigma}(t) x^{\gamma}(t) x^{\gamma \sigma}(t)\left(x^{-\gamma}(t)\right)^{\Delta} .
$$

We claim that

$$
x^{\gamma \sigma}(t)\left(x^{-\gamma}(t)\right)^{\Delta}=-F(w(t), t) .
$$

If $\mu(t)=0$, then

$$
x^{\gamma \sigma}(t)\left(x^{-\gamma}(t)\right)^{\Delta}=-\gamma \frac{x^{\Delta}(t)}{x(t)}=-\gamma\left(\frac{w(t)}{r(t)}\right)^{1 / \gamma}=-F(w(t), t) .
$$

Next assume that $\mu(t)>0$. Then

$$
\begin{aligned}
x^{\gamma \sigma}(t)\left(x^{-\gamma}\right)^{\Delta}(t) & =x^{\gamma \sigma}(t) \frac{\left(x^{-\gamma}\right)^{\sigma}(t)-x^{-\gamma}(t)}{\mu(t)}=-\frac{1}{x^{\gamma}(t)} \frac{x^{\gamma \sigma}(t)-x^{\gamma}(t)}{\mu(t)} \\
& =-\frac{\left(x^{\gamma}\right)^{\Delta}(t)}{x^{\gamma}(t)}=-F(w(t), t)
\end{aligned}
$$

by (2.12). Now by (2.12) and (2.17), we get (2.7). Finally, note that if $x(t) \neq 0$ and $w(t):=$ $r(t)\left(x^{\Delta}(t)\right)^{\gamma / x^{\gamma}}(t)$, then

$$
\frac{x^{\sigma}(t)}{x(t)}=\frac{x(t)+\mu(t) x^{\Delta}(t)}{x(t)}=1+\mu(t) \frac{x^{\Delta}(t)}{x(t)}=1+\mu(t)\left(\frac{w(t)}{r(t)}\right)^{1 / \gamma} .
$$

It follows that $(w(t) / r(t))^{1 / \gamma} \in \mathscr{R}$. Also we get

$$
x(t) x^{\sigma}(t)>0 \quad \text { iff }\left(\frac{w}{r}\right)^{1 / \gamma} \in \mathscr{R}^{+} .
$$

In a similar manner, we may obtain the following.

Theorem 2.2 (factorization of $L_{2}$ ). If $x \in D$ with $x(t) \neq 0$ and $w(t):=r(t)\left(x^{\Delta}(t)\right)^{\gamma} / x^{\gamma}(t)$, then $w \in \mathbb{D}$ and

$$
L_{2} x(t)=x^{\gamma \sigma}(t) R_{2} w(t), \quad t \in \mathbb{T}^{\kappa} .
$$

Conversely, if $w \in \mathbb{D}$ and

$$
x(t):=e_{(w / r)^{1 / \gamma}}\left(t, t_{0}\right),
$$

then $x \in D$ and (2.23) holds. Furthermore, $x(t) x^{\sigma}(t)>0$ if and only if $(w / r)^{1 / \gamma} \in \mathscr{R}^{+}$.

The following corollary follows easily from the factorizations given in Theorems 2.1 and 2.2, respectively, and from the fact that if $x(t) \neq 0$ and $w(t):=r(t)\left(x^{\Delta}(t)\right)^{\gamma} / x^{\gamma}(t)$, then

$$
\frac{x^{\sigma}(t)}{x(t)}=1+\mu(t)\left(\frac{w(t)}{r(t)}\right)^{1 / \gamma}
$$


Corollary 2.3. For $i=1,2$, the following hold.

(a) The dynamic equation $L_{i} x=0$ has a solution $x(t)$ with $x(t) \neq 0$ on $\mathbb{T}$ if and only if the Riccati equation $R_{i} w=0$ has a solution $w(t)$ on $\mathbb{T}^{\kappa}$ with $(w / r)^{1 / \gamma} \in \mathscr{R}$.

(b) The dynamic equation $L_{i} x=0$ has a solution $x(t)$ with $x(t) x^{\sigma}(t)>0$ on $\mathbb{T}$ if and only if the Riccati equation $R_{i} w=0$ has a solution $w(t)$ on $\mathbb{T}^{\kappa}$ with $(w / r)^{1 / \gamma} \in \mathscr{R}^{+}$.

(c) The dynamic inequality $L_{i} x \leq 0$ has a positive solution $x(t)$ on $\mathbb{T}$ if and only if the Riccati inequality $R_{i} z \leq 0$ has a solution $z(t)$ on $\mathbb{T}^{\kappa}$ with $(z / r)^{1 / \gamma} \in \mathscr{R}^{+}$.

We state for convenience the following theorem involving the Riccati technique for equations $L_{1} x=0$ and $L_{2} x=0$. This theorem follows immediately from Theorems 2.1 and 2.2. Part (B) is proven by Řehák [23]. Part (A) is considered by Sun and Li [32] when $\gamma$ is an odd positive integer. The proof of $(\mathrm{A})$ is quite straightforward and is based on an iterative technique. We omit the details.

Theorem 2.4. Assume sup $\mathbb{T}=\infty$ and (1.13) holds.

(A) The Riccati inequality $R_{1} z \leq 0$ has a positive solution on $\left[t_{0}, \infty\right)_{\mathbb{T}}$ if and only if the dynamic equation $L_{1} x=0$ has a positive solution on $\left[t_{0}, \infty\right)_{\mathbb{T}}$.

(B) The Riccati inequality $R_{2} z \leq 0$ has a positive solution on $\left[t_{0}, \infty\right)_{\mathbb{T}}$ if and only if the dynamic equation $L_{2} x=0$ has a positive solution on $\left[t_{0}, \infty\right)_{\mathbb{T}}$.

Theorem 2.5. Assume sup $\mathbb{T}=\infty$ and (1.13) holds.

(A) If $\gamma \geq 1$ and there is $a t_{0} \in[a, \infty)_{\mathbb{T}}$ such that the inequality

$$
z^{\Delta}+p(t)+\frac{\gamma}{r^{1 / \gamma}(t)}\left(1+\mu(t)\left(\frac{z}{r(t)}\right)^{1 / \gamma}\right)^{\gamma-1} z^{(\gamma+1) / \gamma} \leq 0
$$

has a positive solution on $\left[t_{0}, \infty\right)_{\mathbb{T}}$, then $L_{1} x=0$ is nonoscillatory on $[a, \infty)_{\mathbb{T}}$.

(B) If $\gamma \geq 1$ and there exists $a t_{0} \in[a, \infty)_{\mathbb{T}}$ such that the inequality

$$
z^{\Delta}+p(t)+\frac{\gamma}{r^{1 / \gamma}(t)}\left(1+\mu(t)\left(\frac{z}{r(t)}\right)^{1 / \gamma}\right)^{-1} z^{(\gamma+1) / \gamma} \leq 0
$$

has a positive solution on $\left[t_{0}, \infty\right)_{\mathbb{T}}$, then $L_{2} x=0$ is nonoscillatory on $[a, \infty)_{\mathbb{T}}$.

( $\widehat{\mathrm{A}})$ If $0<\gamma \leq 1$ and there is a $t_{0} \in[a, \infty)_{\mathbb{I}}$ such that the inequality

$$
z^{\Delta}+p(t)+\frac{\gamma}{r^{1 / \gamma}(t)} z^{(\gamma+1) / \gamma} \leq 0
$$

has a positive solution on $\left[t_{0}, \infty\right)_{\mathbb{T}}$, then $L_{1} x=0$ is nonoscillatory on $[a, \infty)_{\mathbb{T}}$.

( $\hat{\mathrm{B}})$ If $0<\gamma \leq 1$ and there exists a $t_{0} \in[a, \infty)_{\mathbb{U}}$ such that the inequality

$$
z^{\Delta}+p(t)+\frac{\gamma}{r^{1 / \gamma}(t)}\left(1+\mu(t)\left(\frac{z}{r(t)}\right)^{1 / \gamma}\right)^{-\gamma} z^{(\gamma+1) / \gamma} \leq 0
$$

has a positive solution on $\left[t_{0}, \infty\right)_{\mathbb{T}}$, then $L_{2} x=0$ is nonoscillatory on $[a, \infty)_{\mathbb{T}}$. 
Proof. Assume $\gamma \geq 1$. Using the mean value theorem, one can easily prove that if $x \geq y \geq 0$ and $\gamma \geq 1$, then the inequality

$$
\gamma y^{\gamma-1}(x-y) \leq x^{\gamma}-y^{\gamma} \leq \gamma x^{\gamma-1}(x-y)
$$

holds. We will use (2.30) to show that if $u \geq 0$ and $t \in \mathbb{T}$, then

$$
F(u, t) \leq \gamma\left(1+\mu(t)\left(\frac{u}{r(t)}\right)^{1 / \gamma}\right)^{\gamma-1}\left(\frac{u}{r(t)}\right)^{1 / \gamma} .
$$

For those values of $t \in \mathbb{T}$, where $\mu(t)=0$, it is easy to see that (2.31) is an equality. Now assume $\mu(t)>0$, then using (2.30) we obtain for $u \geq 0$,

$$
F(u, t)=\frac{\left(1+\mu(t)(u / r(t))^{1 / \gamma}\right)^{\gamma}-1}{\mu(t)} \leq \gamma\left(1+\mu(t)\left(\frac{u}{r(t)}\right)^{1 / \gamma}\right)^{\gamma-1}\left(\frac{u}{r(t)}\right)^{1 / \gamma}
$$

and hence (2.31) holds. To prove (A), assume $z$ is a positive solution of $(2.26)$ on $[T, \infty)_{\mathbb{T}}$. Now consider

$$
\begin{aligned}
R_{1} z(t) & =z^{\Delta}(t)+p(t)+z^{\sigma}(t) F(z(t), t) \\
& \leq z^{\Delta}(t)+p(t)+z^{\sigma}(t) \gamma\left(1+\mu(t)\left(\frac{z(t)}{r(t)}\right)^{1 / \gamma}\right)^{\gamma-1}\left(\frac{z(t)}{r(t)}\right)^{1 / \gamma} \quad \text { by }(2.31) \\
& \leq z^{\Delta}(t)+p(t)+z(t) \gamma\left(1+\mu(t)\left(\frac{z(t)}{r(t)}\right)^{1 / \gamma}\right)^{\gamma-1}\left(\frac{z(t)}{r(t)}\right)^{1 / \gamma} \quad \text { by } z^{\Delta}(t) \leq 0 \\
& =z^{\Delta}(t)+p(t)+\gamma\left(1+\mu(t)\left(\frac{z}{r(t)}\right)^{1 / \gamma}\right)^{\gamma-1} \frac{z^{(\gamma+1) / \gamma}(t)}{r^{1 / \gamma}(t)} \leq 0 \quad \text { by }(2.26) .
\end{aligned}
$$

The proof of part (B) of this theorem is very similar, where instead of the inequality (2.31), one uses the inequality

$$
S(u, t) \leq \frac{\gamma}{r^{1 / \gamma}(t)\left(1+\mu(t)(u / r(t))^{1 / \gamma}\right)} u^{(\gamma+1) / \gamma}
$$

for $\gamma \geq 1, u \geq 0, t \in \mathbb{T}$.

Now assume $0<\gamma \leq 1$, then using the mean value theorem, one can show that if $0<$ $y \leq x$, then

$$
\gamma x^{\gamma-1}(x-y) \leq x^{\gamma}-y^{\gamma} \leq \gamma y^{\gamma-1}(x-y)
$$


Using (2.35) we have that for $u \geq 0, t \in \mathbb{T}$,

$$
\begin{gathered}
F(u, t) \leq \gamma\left(\frac{u}{r(t)}\right)^{1 / \gamma}, \\
S(u, t) \leq \frac{\gamma u^{(\gamma+1) / \gamma}}{r^{1 / \gamma}(t)\left(1+\mu(t)(u / r(t))^{1 / \gamma}\right)^{\gamma}} .
\end{gathered}
$$

The rest of the proof for parts $(\widehat{\mathrm{A}})$ and $(\widehat{\mathrm{B}})$ is similar to the proofs for $(\mathrm{A})$ and $(\mathrm{B})$, respectively.

We note that as a special case when $\mathbb{T}=\mathbb{R}$, Theorem 2.5 is related to some results of Li and Yeh [22, Theorem 3.2], Yang [33, Theorem 2], and Yang [33, Corollary 2] for the second-order half-linear differential equation (1.5).

Now, we are ready to establish our main oscillation and nonoscillation results.

Theorem 2.6 (Hille-Kneser-type nonoscillation criteria for $L_{1} x=0$ ). Assume sup $\mathbb{T}=\infty$ and (1.13) holds.

Assume $\gamma \geq 1$. Suppose there exist $a t_{0} \in[a, \infty)_{\mathbb{T}}$, and constants $c \geq 0$, and $d \geq 1$ such that for $t \in\left[t_{0}, \infty\right)_{\mathbb{T}}$,

$$
p(t)+\frac{\gamma c^{(\gamma+1) / \gamma}\left(1+\mu(t)\left(c / t^{d} r(t)\right)^{1 / \gamma}\right)^{\gamma-1}}{\left(t^{d}\right)^{(\gamma+1) / \gamma} r^{1 / \gamma}(t)} \leq \frac{c d}{t(\sigma(t))^{d}} .
$$

Then $L_{1} x=0$ is nonoscillatory on $[a, \infty)_{\mathbb{T}}$. In particular, if for $t \geq t_{0}$ sufficiently large there is a $c \geq 0$ such that

$$
p(t) \leq \frac{c \gamma}{t(\sigma(t))^{\gamma}}\left[1-\left(\frac{c}{r(t)}\right)^{1 / \gamma}\left(\frac{\sigma(t)}{t}\right)^{2 \gamma-1}\right],
$$

then $L_{1} x=0$ is nonoscillatory on $[a, \infty)_{\mathbb{T}}$.

Now assume $0<\gamma \leq 1$. Suppose there exist a $t_{0} \in[a, \infty)_{\mathbb{T}}$, and constants $c \geq 0$, and $0<$ $d \leq 1$ such that for $t \in\left[t_{0}, \infty\right)_{\mathbb{T}}$,

$$
p(t)+\frac{\gamma c^{(\gamma+1) / \gamma}}{\left(t^{d}\right)^{(\gamma+1) / \gamma} r^{1 / \gamma}(t)} \leq \frac{c d}{t^{d} \sigma(t)} .
$$

Then $L_{1} x=0$ is nonoscillatory on $[a, \infty)_{\mathbb{T}}$.

In particular, if for $t \geq t_{0}$ sufficiently large there is a $c \geq 0$ such that

$$
p(t) \leq \frac{c \gamma}{t^{\gamma} \sigma(t)}\left[1-\left(\frac{c}{r(t)}\right)^{1 / \gamma}\left(\frac{\sigma(t)}{t}\right)\right],
$$

then $L_{1} x=0$ is nonoscillatory on $[a, \infty)_{\mathbb{T}}$.

Proof. First assume $\gamma \geq 1$. From Theorem 2.5, we see that if the inequality (2.26) has a positive solution in a neighborhood of $\infty$, then $L_{1} x=0$ is nonoscillatory. Let $z(t):=c / t^{d}$ 
L. Erbe et al. 11

for $t \geq t_{0}$, where $c>0$ and $d \geq 1$. We claim that

$$
z^{\Delta}(t) \leq-\frac{c d}{t(\sigma(t))^{d}}
$$

If $\mu(t)=0$, it is easy to see that (2.41) is an equality. Now assume $\mu(t)>0$, then

$$
z^{\Delta}(t)=\frac{1}{\mu(t)}\left[\frac{c}{(\sigma(t))^{d}}-\frac{c}{t^{d}}\right]=\frac{c}{\sigma(t)-t}\left[\frac{1}{(\sigma(t))^{d}}-\frac{1}{t^{d}}\right]=-\frac{c}{(\sigma(t))^{d} t^{d}}\left(\frac{(\sigma(t))^{d}-t^{d}}{\sigma(t)-t}\right) .
$$

Applying inequality (2.30) to (2.42), we get that

$$
z^{\Delta}(t) \leq-\frac{c}{(\sigma(t))^{d} t^{d}}\left(d t^{d-1}\right)=-\frac{c d}{t(\sigma(t))^{d}} .
$$

Hence (2.41) holds in general. It follows from (2.41) that

$$
\begin{aligned}
z^{\Delta}(t) & +p(t)+\frac{\gamma}{r^{1 / \gamma}(t)}\left(1+\mu(t)\left(\frac{z(t)}{r(t)}\right)^{1 / \gamma}\right)^{\gamma-1} z^{(\gamma+1) / \gamma}(t) \\
& \leq-\frac{c d}{t(\sigma(t))^{d}}+p(t)+\frac{\gamma c^{(\gamma+1) / \gamma}\left(1+\mu(t)\left(c / t^{d} r(t)\right)^{1 / \gamma}\right)^{\gamma-1}}{\left(t^{d}\right)^{(\gamma+1) / \gamma} r^{1 / \gamma}(t)} \leq 0 \quad(\text { by (2.37)) }
\end{aligned}
$$

It then follows from Theorem 2.5 that $L_{1} x=0$ is nonoscillatory on $[a, \infty)_{\mathbb{T}}$.

Letting $d=\gamma$ in (2.37) and simplifying, we have

$$
p(t) \leq \frac{c \gamma}{t(\sigma(t))^{\gamma}}-\frac{\gamma c^{(\gamma+1) / \gamma}}{t^{\gamma+1} r^{1 / \gamma}(t)}\left(1+\frac{\mu(t)}{t r^{1 / \gamma}(t)} c^{1 / \gamma}\right)^{\gamma-1}
$$

Hence, if for some $c \geq 0, p(t)$ satisfies (2.45) for $t \in\left[t_{0}, \infty\right)_{\mathbb{T}}$, then $L_{1} x=0$ is nonoscillatory on $[a, \infty)_{\mathbb{T}}$. Note that since we are assuming $p(t)$ satisfies $(2.38)$ (and $p(t)>0$ ), we have that $c / r(t) \leq 1$ which we use in the next chain of inequalites.

By (2.38),

$$
\begin{aligned}
p(t) & \leq \frac{c \gamma}{t(\sigma(t))^{\gamma}}\left[1-\left(\frac{c}{r(t)}\right)^{1 / \gamma}\left(\frac{\sigma(t)}{t}\right)^{2 \gamma-1}\right]=\frac{c \gamma}{t(\sigma(t))^{\gamma}}-\left(\frac{\gamma c^{(\gamma+1) / \gamma}}{t^{\gamma+1} r^{1 / \gamma}(t)}\right)\left(\frac{\sigma(t)}{t}\right)^{\gamma-1} \\
& =\frac{c \gamma}{t(\sigma(t))^{\gamma}}-\left(\frac{\gamma c^{(\gamma+1) / \gamma}}{t^{\gamma+1} r^{1 / \gamma}(t)}\right)\left(1+\frac{\mu(t)}{t}\right)^{\gamma-1} \\
& \leq \frac{c \gamma}{t(\sigma(t))^{\gamma}}-\left(\frac{\gamma c^{(\gamma+1) / \gamma}}{t^{\gamma+1} r^{1 / \gamma}(t)}\right)\left(1+\frac{\mu(t)}{t}\left(\frac{c}{r(t)}\right)^{1 / \gamma}\right)^{\gamma-1} .
\end{aligned}
$$

Hence (2.45) holds and thus $L_{1} x=0$ is nonoscillatory on $[a, \infty)_{\mathbb{T}}$. 
To prove the second half of this theorem (the case $0<\gamma \leq 1$ ) note that from (2.35) in this case ( since $0<d \leq 1$ ), one gets the inequality

$$
z^{\Delta}(t) \leq-\frac{c d}{t^{d} \sigma(t)}
$$

instead of (2.41). The proof of the result concerning (2.39) follows directly from $(\widehat{A})$ in Theorem 2.5 and the result concerning (2.40) follows easily by letting $d=\gamma$ in (2.39).

Similar to the proof of Theorem 2.6, one can establish the following result.

Theorem 2.7 (Hille-Kneser-type nonoscillation criteria for $L_{2} x=0$ ). Assume sup $\mathbb{T}=\infty$ and (1.13) holds.

Assume $\gamma \geq 1$. If for $t \geq t_{0}$ sufficiently large, there exist positive constants $c$ and $d \geq 1$ such that

$$
p(t)+\frac{\gamma c^{(\gamma+1) / \gamma}}{\left(t^{d}\right)^{(\gamma+1) / \gamma} r^{1 / \gamma}(t)\left(1+\mu(t)\left(c / t^{d} r(t)\right)^{1 / \gamma}\right)} \leq \frac{c d}{t(\sigma(t))^{d}}
$$

then $L_{2} x=0$ is nonoscillatory on $[a, \infty)_{\mathbb{T}}$. In particular, if for $t \geq t_{0}$ sufficiently large there is $a c \geq 0$ such that

$$
p(t) \leq \frac{c \gamma}{t(\sigma(t))^{\gamma}}\left[1-\left(\frac{\sigma(t)}{t}\right)^{\gamma}\left(\frac{(c / r(t))^{1 / \gamma}}{1+(\mu(t) / t)(c / r(t))^{1 / \gamma}}\right)\right],
$$

then $L_{2} x=0$ is nonoscillatory on $[a, \infty)_{\mathbb{T}}$.

Now assume $0<\gamma \leq 1$. If for $t \geq t_{0}$ sufficiently large there exist positive constants $c$ and $0<d \leq 1$ such that

$$
p(t)+\frac{\gamma c^{(\gamma+1) / \gamma}}{\left(t^{d}\right)^{(\gamma+1) / \gamma} r^{1 / \gamma}(t)\left(1+\mu(t)\left(c / t^{d} r(t)\right)^{1 / \gamma}\right)^{\gamma}} \leq \frac{c d}{t^{d} \sigma(t)},
$$

then $L_{2} x=0$ is nonoscillatory on $[a, \infty)_{\mathbb{T}}$. In particular, if for $t \geq t_{0}$ sufficiently large there is $a c \geq 0$ such that

$$
p(t) \leq \frac{c \gamma}{t^{\gamma} \sigma(t)}\left[1-\left(\frac{\sigma(t)}{t}\right)\left(\frac{(c / r(t))^{1 / \gamma}}{\left(1+(\mu(t) / t)(c / r(t))^{1 / \gamma}\right)^{\gamma}}\right)\right],
$$

then $L_{2} x=0$ is nonoscillatory on $[a, \infty)_{\mathbb{T}}$.

We now give some interesting examples.

Example 2.8. If $\mathbb{T}=\mathbb{R}$, then $L_{1} x=0$ and $L_{2} x=0$ are the same. If $\gamma=1$, the conditions (2.38) and (2.49) both reduce to

$$
p(t) \leq \frac{c}{t^{2}}\left(1-\frac{c}{r(t)}\right)
$$


In the special case $r(t) \equiv 1$, this reduces (taking $c=1 / 2$ ) to

$$
p(t) \leq \frac{1}{4 t^{2}}
$$

which is the Hille-Kneser criterion mentioned in Theorem 1.1.

More generally, if $\gamma>0$ and $r(t) \equiv 1$, then (2.38) and (2.40) with $c=(\gamma /(\gamma+1))^{\gamma}$ both reduce to

$$
p(t) \leq\left(\frac{\gamma}{\gamma+1}\right)^{\gamma+1} \frac{1}{t^{1+\gamma}}
$$

Moreover, in the case $\gamma>1$, Došlý and Řehák [11] have improved this criterion.

Example 2.9. If $\mathbb{T}=\mathbb{N}, \gamma=1$, and $r(t) \equiv 1$, then the condition (2.38) for $L_{1} x=0$ reduces to

$$
p(t) \leq \frac{c}{t(t+1)}\left(1-c \frac{t+1}{t}\right)
$$

Letting $c=1 / 2$, it is easily seen that if there is a $k<1 / 4$ such that

$$
p(t) \leq \frac{k}{t(t+1)}
$$

for large $t$, then $L_{1} x=0$ is nonoscillatory on $\mathbb{N}$.

If $\mathbb{T}=\mathbb{N}, r(t) \equiv 1, \gamma=1$, condition (2.49) reduces to

$$
p(t) \leq \frac{c}{t(t+1)} \frac{1-c}{1+c / t}
$$

Letting $c=1 / 2$, one can argue that if there is a $k<1 / 4$ such that

$$
p(t) \leq \frac{k}{t(t+1)}
$$

for large $t$, then $L_{2} x=0$ is nonoscillatory on $\mathbb{N}$.

If $\gamma \geq 1, r(t) \equiv 1$, then using (2.49) it is not difficult to see that if there exists $k<$ $(\gamma /(\gamma+1))^{\gamma+1}$ such that

$$
p(t) \leq \frac{k}{t(t+1)^{\gamma}}
$$

for large $t$, then $L_{2} x=0$ is nonoscillatory on $\mathbb{N}$. On the other hand, if $0<\gamma \leq 1$, then using (2.51), it is easily shown that if there exists $k<(\gamma /(\gamma+1))^{\gamma+1}$ such that

$$
p(t) \leq \frac{k}{t^{\gamma}(t+1)}
$$


for large $t$, then $L_{2} x=0$ is nonoscillatory on $\mathbb{N}$. Combining these results, we see that if $\gamma>0, r(t) \equiv 1$, and there is a $k<(\gamma /(\gamma+1))^{\gamma+1}$ such that

$$
p(t) \leq \frac{k}{t^{\gamma+1}}
$$

for large $t$, then $L_{2} x=0$ is nonoscillatory on $\mathbb{N}$. See Agarwal et al. [1] for additional results.

Example 2.10. If $\mathbb{T}=q^{N_{0}}, q>1$, then (2.38) becomes (in the case $\gamma=1$ and $r(t) \equiv 1$ )

$$
p(t) \leq \frac{c}{q t^{2}}(1-c q)
$$

Taking $c=1 / 2 q$, we get

$$
p(t) \leq \frac{1}{4 q^{2} t^{2}}
$$

for large $t$ implies $L_{1} x=0$ is nonoscillatory on $q^{\mathbb{N}_{0}}$. With the same assumptions $(\mathbb{T}=$ $q^{\mathbb{N}_{0}}, q>1, r(t) \equiv 1, \gamma=1$ ), condition (2.49) becomes

$$
p(t) \leq \frac{c}{q t^{2}} \frac{1-c}{1+(q-1) c}
$$

and with $c=1 /(1+\sqrt{q})$, we get

$$
p(t) \leq \frac{1}{q(1+\sqrt{q})^{2} t^{2}}
$$

for large $t$ implies the nonoscillation of $L_{2} x=0$ on $q^{\mathbb{N}_{0}}$.

We see therefore that the criteria for nonoscillation of the linear $(\gamma=1)$ equations $L_{1} x=0$ and $L_{2} x=0$ as consequences of Theorems 2.6 and 2.7 are different in general. Solving the Euler-Cauchy equations

$$
\begin{aligned}
& x^{\Delta \Delta}+\frac{a}{t \sigma(t)} x=0, \\
& x^{\Delta \Delta}+\frac{a}{t \sigma(t)} x^{\sigma}=0,
\end{aligned}
$$

one can show that if $a \leq 1 / 4$, then (2.66) is nonoscillatory on $q^{\mathbb{N}_{0}}$, and if $a \leq 1 /(\sqrt{q}+1)^{2}$, then (2.67) is nonoscillatory on $q^{\mathbb{N}_{0}}$. We note that the result (2.63) is not sharp; however, the result (2.65) is sharp as can be seen by a more detailed analysis. See also Rehák [23].

If $\gamma \geq 1, r(t) \equiv 1$, then applying (2.38), we get that if

$$
p(t) \leq \frac{1}{q^{2 \gamma^{2}}}\left(\frac{\gamma}{\gamma+1}\right)^{\gamma+1} \frac{1}{t^{1+\gamma}}
$$


for large $t$, then $L_{1} x=0$ is nonoscillatory. On the other hand, if $0<\gamma \leq 1$, then applying (2.40), we get that if

$$
p(t) \leq \frac{1}{q^{\gamma+1}}\left(\frac{\gamma}{\gamma+1}\right)^{\gamma+1} \frac{1}{t^{1+\gamma}}
$$

for large $t$, then $L_{1} x=0$ is nonoscillatory. If $\gamma \geq 1, r(t) \equiv 1$, we get using (2.49) that if

$$
p(t) \leq \frac{c \gamma}{t^{1+\gamma} q^{\gamma}}\left[1-q^{\gamma}\left(\frac{c^{1 / \gamma}}{1+(q-1) c^{1 / \gamma}}\right)\right]
$$

for large $t$, then $L_{2} x=0$ is nonoscillatory. On the other hand, if $0<\gamma \leq 1$, then using (2.51), we get that if

$$
p(t) \leq \frac{c \gamma}{t^{1+\gamma} q^{\gamma}}\left[1-q^{\gamma}\left(\frac{c^{1 / \gamma}}{\left[1+(q-1) c^{1 / \gamma}\right]^{\gamma}}\right)\right]
$$

for large $t$, then $L_{2} x=0$ is nonoscillatory.

Example 2.11. For a general time scale $\mathbb{T}$, where sup $\mathbb{T}=\infty$ and (1.13) holds, it follows from (2.38) that if $\gamma>1$, and

$$
\left(\frac{\sigma(t)}{t}\right)^{2 \gamma-1} \leq m r^{1 / \gamma}(t)
$$

for large $t$, for some constant $m>0$, then $L_{1} x=0$ is nonoscillatory on $[a, \infty)_{\mathbb{T}}$ provided

$$
t(\sigma(t))^{\gamma} p(t) \leq m\left(\frac{\gamma}{m(\gamma+1)}\right)^{\gamma+1}
$$

for large $t$. To see this, observe that in (2.38) the right-hand side is bounded above by

$$
\frac{\gamma}{t(\sigma(t))^{\gamma}} h(c)
$$

where $h(c):=c-c^{(\gamma+1) / \gamma} m$, which has its maximum at $c=(\gamma / m(\gamma+1))^{\gamma}$. (For $\mathbb{T}=\mathbb{R}$, $\gamma=1, r(t) \equiv 1$, we can take $m=1$ and this again reduces to the Hille-Kneser criterion.)

For the case of $L_{2} x=0$, we first observe that in (2.49), the expression

$$
\frac{(c / r(t))^{1 / \gamma}}{1+(\mu(t) / t)(c / r(t))^{1 / \gamma}} \leq\left(\frac{c}{r(t)}\right)^{1 / \gamma}
$$


so that the right-hand side of (2.49) is bounded below by

$$
\frac{c \gamma}{t(\sigma(t))^{\gamma}}\left[1-\left(\frac{\sigma(t)}{t}\right)^{\gamma}\left(\frac{c}{r(t)}\right)^{1 / \gamma}\right] \text {. }
$$

Therefore, if there exists an $m>0$ such that

$$
\left(\frac{\sigma(t)}{t}\right)^{\gamma} \leq m r^{1 / \gamma}(t)
$$

for large $t$, then $L_{2} x=0$ is nonoscillatory provided (2.73) holds. Notice that if $\gamma=1$, $\mathbb{T}=\mathbb{R}, r(t) \equiv 1$, then (2.73) reduces to the Hille-Kneser criterion $p(t) \leq 1 / 4 t^{2}$. One can also give additional special cases. We leave this to the interested reader.

\section{Acknowledgment}

The authors gratefully acknowledge the referee's detailed comments on and corrections for an earlier version.

\section{References}

[1] R. P. Agarwal, M. Bohner, S. R. Grace, and D. O’Regan, Discrete Oscillation Theory, Hindawi, New York, 2005.

[2] R. P. Agarwal, M. Bohner, and S. H. Saker, Oscillation of second order delay dynamic equations, to appear in The Canadian Applied Mathematics Quarterly.

[3] E. Akin-Bohner, M. Bohner, and S. H. Saker, Oscillation criteria for a certain class of second order Emden-Fowler dynamic equations, to appear in Electronic Transactions on Numerical Analysis.

[4] E. Akin-Bohner and J. Hoffacker, Oscillation properties of an Emden-Fowler type equation on discrete time scales, Journal of Difference Equations and Applications 9 (2003), no. 6, 603-612.

[5] M. Bohner and A. Peterson, Dynamic Equations on Time Scales, Birkhäuser, Massachusetts, 2001.

[6] M. Bohner and A. Peterson (eds.), Advances in Dynamic Equations on Time Scales, Birkhäuser, Massachusetts, 2003.

[7] M. Bohner and S. H. Saker, Oscillation criteria for perturbed nonlinear dynamic equations, Mathematical and Computer Modelling, Boundary Value Problems and Related Topics 40 (2004), no. 3-4, 249-260.

[8] Oscillation of second order nonlinear dynamic equations on time scales, The Rocky Mountain Journal of Mathematics 34 (2004), no. 4, 1239-1254.

[9] X. C. Cai, An existence theorem for second order discrete boundary value problems, Mathematics in Economics 22 (2005), no. 2, 208-214.

[10] O. Došlý and S. Hilger, A necessary and sufficient condition for oscillation of the Sturm-Liouville dynamic equation on time scales, Journal of Computational and Applied Mathematics 141 (2002), no. 1-2, 147-158, special issue on "Dynamic Equations on Time Scales", edited by R. P. Agarwal, M. Bohner, and D. O'Regan.

[11] O. Došlý and P. Řehák, Half-Linear Differential Equations, North-Holland Mathematics Studies, vol. 202, Elsevier Science B.V., Amsterdam, 2005. 
[12] L. Erbe, Oscillation criteria for second order linear equations on a time scale, The Canadian Applied Mathematics Quarterly 9 (2001), no. 4, 345-375 (2002).

[13] L. Erbe and A. Peterson, Riccati equations on a measure chain, Dynamic Systems and Applications, Vol. 3 (Atlanta, GA, 1999) (G. S. Ladde, N. G. Medhin, and M. Sambandham, eds.), Dynamic, Georgia, 2001, pp. 193-199.

[14] Oscillation criteria for second-order matrix dynamic equations on a time scale, Journal of Computational and Applied Mathematics 141 (2002), no. 1-2, 169-185, special issue on "Dynamic Equations on Time Scales", edited by R. P. Agarwal, M. Bohner, and D. O'Regan.

[15] _ Boundedness and oscillation for nonlinear dynamic equations on a time scale, Proceedings of the American Mathematical Society 132 (2004), no. 3, 735-744.

[16] L. Erbe, A. Peterson, and S. H. Saker, Oscillation criteria for second-order nonlinear dynamic equations on time scales, Journal of the London Mathematical Society 67 (2003), no. 3, 701-714.

[17] _ Asymptotic behavior of solutions of a third-order nonlinear dynamic equation on time scales, Journal of Computational and Applied Mathematics 181 (2005), no. 1, 92-102.

[18] G. Sh. Guseinov and B. Kaymakçalan, On a disconjugacy criterion for second order dynamic equations on time scales, Journal of Computational and Applied Mathematics 141 (2002), no. 1-2, 187-196, special issue on "Dynamic Equations on Time Scales", edited by R. P. Agarwal, M. Bohner, and D. O’Regan.

[19] S. Hilger, Analysis on measure chains - a unified approach to continuous and discrete calculus, Results in Mathematics 18 (1990), no. 1-2, 18-56.

[20] E. Hille, Non-oscillation theorems, Transactions of the American Mathematical Society 64 (1948), 234-252.

[21] T. Kusano and N. Yoshida, Nonoscillation theorems for a class of quasilinear differential equations of second order, Journal of Mathematical Analysis and Applications 189 (1995), no. 1, 115-127.

[22] H. J. Li and C. C. Yeh, Sturmian comparison theorem for half-linear second-order differential equations, Proceedings of the Royal Society of Edinburgh. Section A. Mathematics 125 (1995), no. 6, 1193-1204.

[23] P. Řehák, Half-linear dynamic equations on time scales: IVP and oscillatory properties, Nonlinear Functional Analysis and Applications 7 (2002), no. 3, 361-403.

[24] _ Half-linear dynamic equations on time scales, Habilitation thesis, Masaryk University, Brno, 2005.

[25] _ How the constants in Hille-Nehari theorems depend on time scales, Advances in Difference Equations 2006 (2006), Article ID 64534, 15 pages.

[26] S. H. Saker, Oscillation of nonlinear dynamic equations on time scales, Applied Mathematics and Computation 148 (2004), no. 1, 81-91.

[27] __ Oscillation criteria of second-order half-linear dynamic equations on time scales, Journal of Computational and Applied Mathematics 177 (2005), no. 2, 375-387.

[28] - Oscillation of second-order nonlinear neutral delay dynamic equations on time scales, Journal of Computational and Applied Mathematics 187 (2006), no. 2, 123-141.

[29] _ Boundedness of solutions of second-order forced nonlinear dynamic equations, to appear in The Rocky Mountain Journal of Mathematics.

[30] _ New oscillation criteria for second-order nonlinear dynamic equations on time scales, to appear in Nonlinear Functional Analysis and Applications.

[31] J. Sugie, K. Kita, and N. Yamaoka, Oscillation constant of second-order non-linear self-adjoint differential equations, Annali di Matematica Pura ed Applicata 181 (2002), no. 3, 309-337.

[32] H.-R. Sun and W.-T. Li, Positive solutions of second-order half-linear dynamic equations on time scales, Applied Mathematics and Computation 158 (2004), no. 2, 331-344. 


\section{Hille-Kneser-type criteria}

[33] X. Yang, Nonoscillation criteria for second-order nonlinear differential equations, Applied Mathematics and Computation 131 (2002), no. 1, 125-131.

L. Erbe: Department of Mathematics, University of Nebraska-Lincoln, Lincoln, NE 68588-0130, USA

E-mail address: lerbe@math.unl.edu

A. Peterson: Department of Mathematics, University of Nebraska-Lincoln, Lincoln, NE 68588-0130, USA

E-mail address: apeterso@math.unl.edu

S. H. Saker: Department of Mathematics, Faculty of Science, Mansoura University, Mansoura 35516, Egypt

E-mail address: shsaker@mans.edu.eg 